\title{
Fin du Pléistocène et début de l'Holocène dans la vallée du Jabron (Var, France) : les occupations humaines à la Baume de Monthiver
}

\author{
Guillaume Porraz, Antonin Tomasso, Carlo Mologni, Benjamin Audiard, Leïla Hoareau, \\ Marie-Anne Julien, Marco Padovan, Giorgia Sardelli, Patrick Simon, Louise Purdue
}

Une suite de prospections consacrées à l'arc de Castellane dans le nord du département du Var a conduit en 2012 à la découverte du site des Prés de Laure (Porraz et al., 2014), positionné en amont de la moyenne vallée du Jabron (Costa et al., 2015). En 2016, un premier travail de recensement et de formalisation des données culturelles et naturelles a été entrepris à l'échelle de cette moyenne vallée et de ses alentours proches (Porraz et al., 2016). Ce programme a permis de procéder à des prospections géomorphologiques et des carottages mécanisés, d'identifier de nouveaux sites préhistoriques en plein air et de découvrir, autour de la vallée, des premiers sites en grottes et abris.

Parmi ces derniers, la Baume de Monthiver a retenu notre attention en raison de la présence en surface d'un matériel archéologique exposé suite à une activité clandestine. La découverte de silex taillés, de restes fauniques et d'un élément de parure suggérait une occupation humaine de la fin du Paléolithique supérieur. Cette note rend compte d'une opération de sondage menée au cours de l'été 2017.

Le site s'ouvre au sud-est le long de la falaise des Baumes sur le massif jurassique de Monthiver à une altitude d'environ 900 m, au-dessus du vallon de la Montade et du site des Prés de Laure (fig. 1A). L'abri se présente comme un petit porche d'environ $5 \mathrm{~m}$ de large pour $10 \mathrm{~m}$ de profondeur (fig. 1B). Le dégagement des déblais remaniés a permis de mettre au jour les limites d'une fouille clandestine prenant la forme d'une tranchée d'orientation est-ouest. La présence d'une discontinuité dans l'agencement des dépôts (fig. 1C) nous a encouragé à ouvrir deux secteurs (fig. 1B) : l'un sous la forme d'un sondage profond (sondage '12'), l'autre sous la forme d'une fouille plus extensive (secteur ' $\mathrm{G}-\mathrm{F}$ ').

Notre intervention a permis de dégager une séquence sédimentaire d'environ $90 \mathrm{~cm}$ d'épaisseur jusqu'au substrat rocheux (fig. 1D). Cette séquence a été subdivisée en sept phases sédimentaires (de A à G) comprenant 23 unités stratigraphiques (US). Les processus de formation du site montrent l'importance des phénomènes de ruissellement, d'érosion et de cryoclastie. Schématiquement, le comblement de l'abri se comprend en trois étapes principales qui marquent une réorientation dans la géométrie des dépôts, initialement de pendage nord (phases sédimentaires $\mathrm{G}$ à $\mathrm{D}$ ), puis ouest (phases $\mathrm{C}$ à $\mathrm{B}$ ) avant un dernier comblement plus régulier à l'horizontal (phase $\mathrm{A}$ ). Les premières analyses géoarchéologiques témoignent d'US fortement remobilisées et soulignent une contribution substantielle des témoins de combustion dans la partie supérieure des dépôts, ce que confirment les valeurs de susceptibilité magnétique.
Le matériel archéologique comprend essentiellement des silex taillés auxquels s'ajoutent quelques nodules ferrugineux isolés ainsi que des restes fauniques, malacologiques et anthracologiques. Une date ${ }^{14} \mathrm{C}$ sur charbon positionne la partie inférieure de la Baume (US M-E21) au cours du Tardiglacaire, dans le Dryas ancien (14 millénaire cal $\mathrm{BP})$, tandis que deux dates ${ }^{14} \mathrm{C}$ sur os calent la partie supérieure (US M-B'3) au début de l'Holocène, dans le Boréal ( $9^{\mathrm{e}}$ millénaire cal BP, fig. 1E).

La séquence archéologique se caractérise par deux principaux ensembles d'occupations séparés par une phase sédimentaire stérile (D). Ces deux ensembles se distinguent par leur densité en vestiges, les états de surface du matériel et la nature des traditions techniques. L'ensemble inférieur (US M-E21) est représenté par quatre éléments laminaires réguliers en silex du Valanginien présentant un bon état de surface. Le profil technoéconomique de ce petit assemblage (produits finis homogènes d'un point de vue technique et pétrographique, absence de petits éléments taillés et de déchets de taille) appuierait l'hypothèse d'une courte incursion au cours du Paléolithique supérieur. Ces éléments ont été retrouvés à proximité d'une petite structure circulaire limoneuse à rares microcharbons (dont Pinus sylvestris/nigra), probablement d'origine anthropique.

L'ensemble archéologique supérieur constitue le cœur des occupations humaines et recouvre trois phases sédimentaires (C à $\mathrm{A}$ ) livrant un total de 626 silex taillés fortement altérés (patines, désilicification, dommages thermiques, ébréchures) et répartis sans discontinuité stratigraphique sur une dizaine d'US. Les systèmes de production montrent une recherche de produits lamino-lamellaires peu réguliers selon des modalités unidirectionnelles et une technique de percussion directe à la pierre ; le spectre des matières premières est strictement $d$ 'origine locale avec une forte représentation des formations d'âge tertiaire ; le seul élément retouché identifié (refus de tamis en cours de tri) est représenté par un fragment de pièce à dos (fig. 1F).

Cet ensemble archéologique supérieur comprend aussi quelques restes fauniques très fragmentés et en général indéterminables, mais les observations préliminaires sur le matériel remanié ont permis de reconnaitre la présence de mammifères de taille petite à moyenne (lièvre et petits artiodactyles). À ce corpus faunique il faut associer la découverte de plusieurs restes de malacofaune terrestre (d'origine naturelle) et marine (d'origine anthropique). Ces derniers $(\mathrm{n}=7)$ appartiennent à l'espèce Columbella rustica et portent les traces d'une perforation émoussée et de résidus de matériaux colorants qui suggèrent leur utilisation en éléments de parure. 

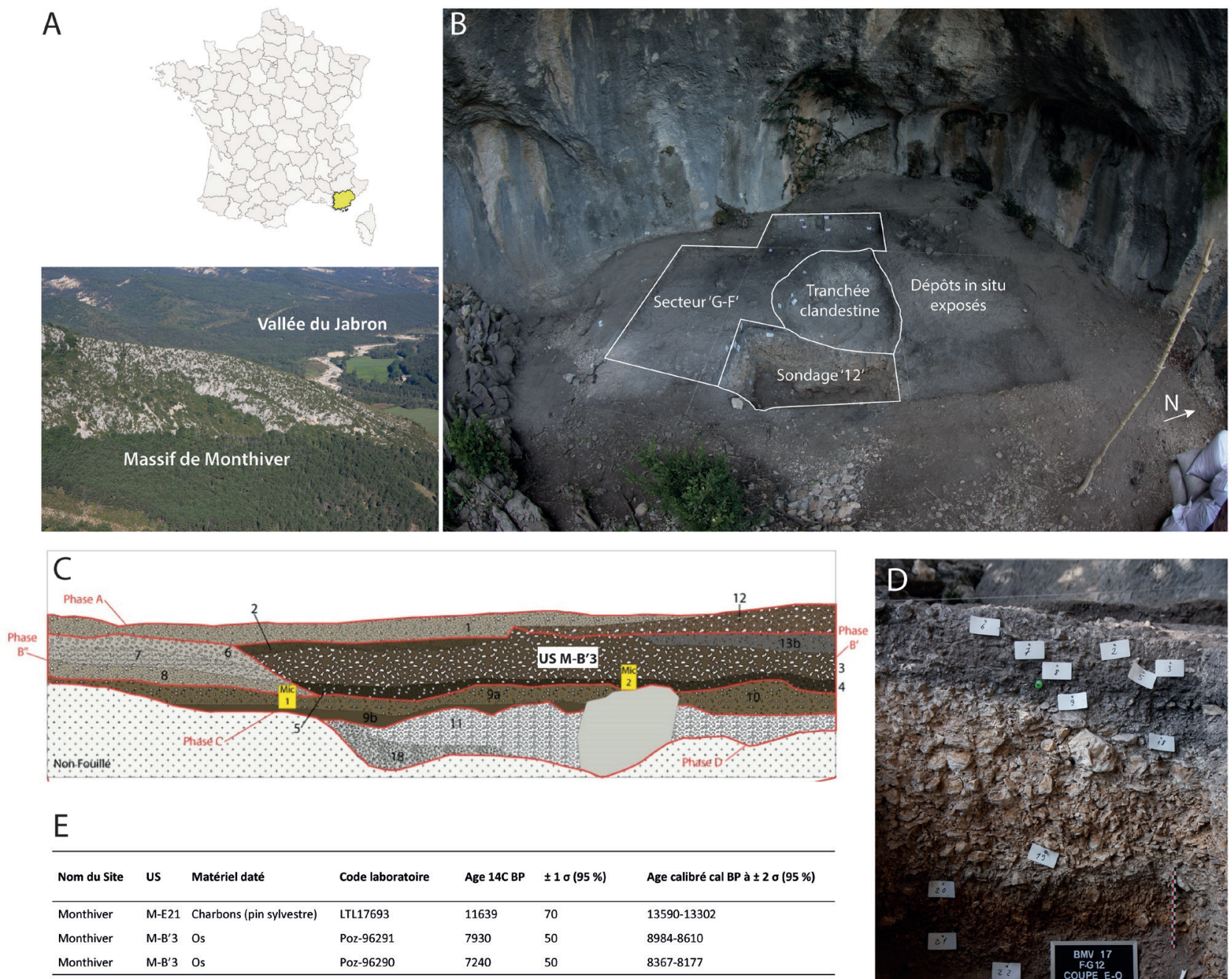

Rq: Les dates ont été calibrées par le programme OXCAL version 4.3 selon IntCal13 (Reimer et al. 2013)

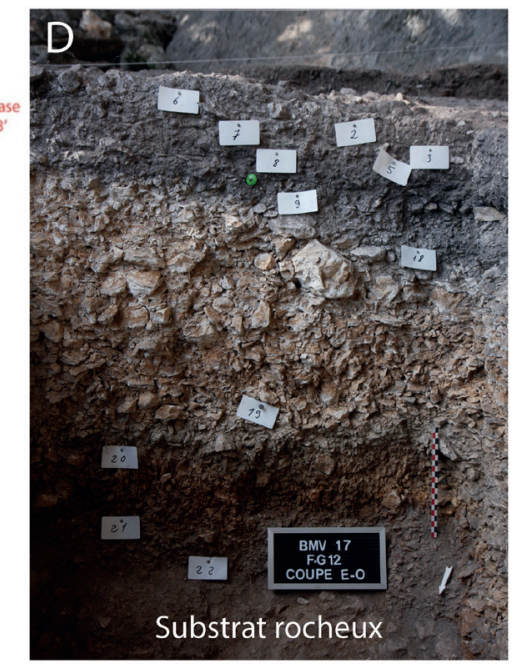

$\mathrm{F}$

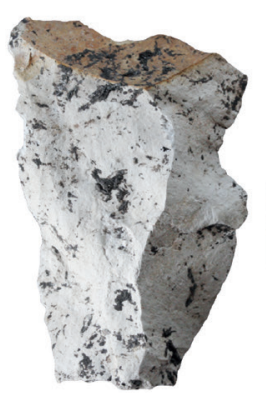

BMV17_\#697_US M-B'3

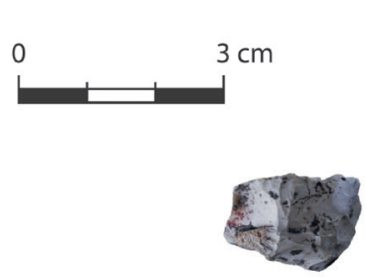

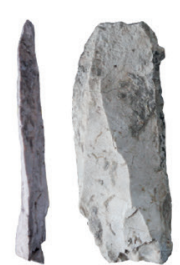

BMV17_\#393_US M-B'3

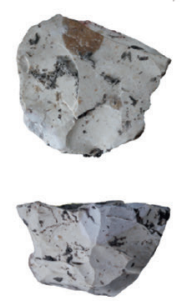

BMV17_\#787_US M-B'3
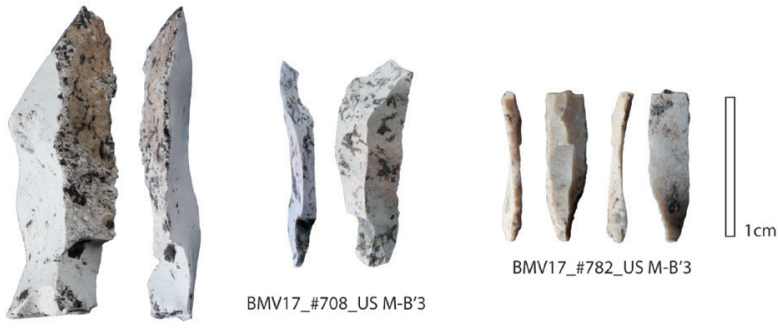

BMV17_\#708_US M-B'3

BMV17_\#690_US M-B'3

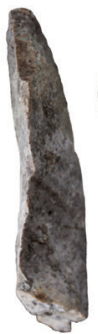

BMV17_\#667_US M-B'3
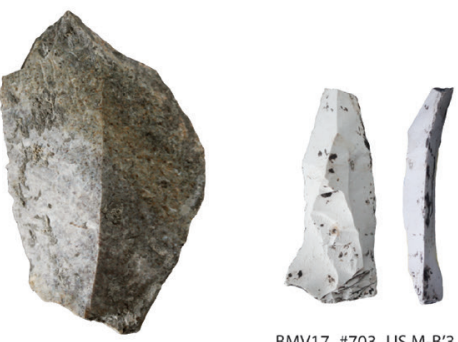

BMV17_\#703_US M-B'3

Fig. 1 - A, vue nord-ouest sur le massif de Monthiver avec la vallée du Jabron en arrière-plan ; B, photographie et planimétrie de la Baume de Monthiver en fin de fouille; $\mathbf{C}$, coupe sagittale redressée de la tranchée clandestine avant fouille (avec localisation de l'US M-B'3) ; D, coupe sagittale du sondage '12' (avec localisation de I'US M-E21 à la base) ; E, dates radiocarbones calibrées (cal BP) ; $\mathbf{F}$, éléments lamino-lamellaires de l'US M-B'3 (\#782 à l'échelle X2). 
La Baume de Monthiver ouvre une nouvelle fenêtre chronologique et fonctionnelle sur l'occupation humaine des Préalpes de Castellane entre la toute fin du Paléolithique supérieur et le premier Mésolithique. De nombreuses questions restent en suspens : registres de subsistance et activités humaines, nature des signatures paléo-environnementales, importance des (hyper)microlithes au sein des assemblages lithiques. Les dates de la phase supérieure de la Baume de Monthiver sont comparables à celles de plusieurs sites régionaux attribués au Sauveterrien (Binder, 1987 ; Livache et Brochier, 2004), dont celui des Agnels dans le Vaucluse (Guilbert, 2003), ou encore de la Baume Fontbrégoua (Courtin, 1975) et de l'abri Saint-Mître dans les Alpes-de-Haute-Provence (Onoratini, 1977). Les occupations de l'abri Martin (Alpes-Maritimes), situé à $40 \mathrm{~km}$ à l'est de la Baume de Monthiver, renseignent quant à elles des occupations un peu plus anciennes (ca. 11000 cal. BP ; Tomasso et $a l ., 2014$ ) qui pourraient partiellement recouvrir les premières occupations humaines enregistrées dans l'abri.

La découverte de la Baume de Monthiver permet de réengager la question des formes et contextes d'émergence des premières sociétés mésolithiques dans le sud-est de la France. Ce contexte régional est sensible d'un point de vue historique puisqu'il se positionne à l'interface de traditions techniques différentes (Tomasso, 2016). Ainsi la fin du Paléolithique supérieur se caractérise-t-elle à l'ouest par des traditions aziliennes puis laboriennes et épilaboriennes (Langlais et al., 2014) tandis qu'à l'est perdurent des traditions techniques de l'Épigravettien (Naudinot et al., 2017). La caractérisation des scénarios d'émergence et des premières expressions du Sauveterrien, côté français et italien (Valdeyron, 2008 ; Visentin, 2017), demandent aujourd'hui une bonne maitrise chronologique et paléoclimatique de la fin du Paléolithique supérieur et du début de l'Holocène dans l'espace nord-méditerranéen.

La Baume de Monthiver apporte son lot de nouvelles archives naturelles et culturelles et vient enrichir un terrain de recherche - la moyenne vallée du Jabron - qui est amené à tenir une place centrale à l'échelle régionale. Cette échelle est celle de l'arc Liguro-Provençal, couloir de circulations, de transferts et de contacts au sein d'un bassin méditerranéen qui se distingue volontiers dans son espace continental (Tomasso et al., 2016). À l'instar du complexe des Balzi Rossi et de la grotte Cosquer, la vallée du Jabron se présente comme un nouveau lieu de mémoire durablement occupé au cours de la préhistoire.

Remerciements : Cette opération a été soutenue par le ministère de la Culture, DRAC PACA et la commune de Compssur-Artuby. Un grand merci à C. Rouvier, inventeur du site, à M. Robert, propriétaire, et aux habitants de Jabron. Ce projet s'inscrit dans le cadre d'une collaboration avec le Parc Naturel Régional du Verdon, le Musée départemental des Gorges du Verdon (Quinson), le Musée d'Anthropologie Préhistorique de Monaco ainsi que les municipalités de Comps-sur-Artuby et de Trigance.

\section{RÉFÉRENCES BIBLIOGRAPHIQUES}

BINDER D. (1987) - Le Néolithique ancien provençal : typologie et technologie des outillages lithiques, vol. 1, Gap, édition du CNRS (coll. Supplément à Gallia Préhistoire, 24), $205 \mathrm{p}$.

Brochier J.-E., Livache M. (2004) - Les traces des derniers chasseurs cueilleurs, in J. Buisson-Catil, A. Guilcher, C. Hussy, M. Olive, et M. Pagni (dir.), Vaucluse préhistorique: le territoire, les hommes, les cultures et les sites, Alain Barthelemy, p. 111-126.

Costa S., Davtian G., Purdue L., Tomasso A., Porraz G. (2015) - Cartographie géomorphologique en contexte archéologique dans la moyenne vallée du Jabron (Var, France), Géomatique Expert, 107, p. 20-31.

Courtin J. (1975) - Le mésolithique de la Baume Fontbrégoua à Salerne (Var), Cahiers ligures de préhistoire et d'archéologie, 24, p. 110-117.

Guilbert R. (2003) - Les systèmes de débitage de trois sites sauveterriens dans le Sud-Est de la France, Bulletin de la Société préhistorique française, 100, 3, p. 463-478.

Langlais M., Bonnet-Jacquement P., Detrain L., ValdeyRON N. (2014) - Le Laborien : ultime sursaut technique du cycle évolutif paléolithique du Sud-Ouest de la France?, in J. Jaubert, N. Fourment, et P. Depaepe (dir.), Transition, ruptures et continuités durant la Préhistoire -2- Le Paléolithique et le Mésolithique, Paris, Société Préhistorique Française, p. 567-583.

Naudinot N., Tomasso A., Messager E., Finsinger W., Ruffaldi P., Langlais M. (2017) - Between Atlantic and Mediterranean: Changes in technology during the Late Glacial in Western Europe and the climate hypothesis, Quaternary International, 428, p. 33-49.

OnORATINI G. (1977) - Un faciès provençal du Sauveterrien : l'abri Saint-Mître à Reillanne (Alpes-de-Haute-Provence), Congrès préhistorique de France, Compte rendu de la XX session, Provence, $1^{\text {er }}$-7 juillet 1974, Issoudun, Imprimerie Laboureur et Cie, p. 391-398.

Porraz G., Tomasso A., Purdue L. (2014) - Les Prés-deLaure : un premier site du Paléolithique supérieur sur les terrasses de la moyenne vallée du Jabron (Var, France), Bulletin de la Société préhistorique française, 111, 1, p. 135138.

Porraz G., Purdue L., Costa S., Garcin P., Hoareau L., Julien M-A., Sorin S., Tomasso A. (2016) - Une préhistoire de la moyenne vallée du Jabron (Communs de Compssur-Artuby, Trigance, Brenon et Bargème, Var), Service régional de l'archéologie Provence-Alpes-Côtes-d'Azur, $123 \mathrm{p}$.

Tomasso A. (2016) - Une unité de façade. Évolution des systèmes techniques tardiglaciaires entre l'Allerød et le Dryas récent en Europe méditerranéenne, Bulletin de la Société préhistorique française, 113, 2, p. 241-264.

Tomasso A., Naudinot N., Binder D., Grimaldi S. (2014) Unité et diversité dans l'Épigravettien récent de l'arc liguroprovençal, in M. Langlais, N. Naudinot et M. Peresani (dir.), Les groupes culturels de la transition Pléistocène-Holocène 
entre Atlantique et Adriatique, Paris, Société préhistorique française (coll. Séances, 3), p. 155-185.

Tomasso A., Binder D., Martino G., Porraz G., Simon P. (2016) - Le référentiel Matières Premières de l'Arc LiguroProvençal (MP-ALP) : ressources siliceuses entre vallée du Rhône et Apennins, in A. Tomasso, D. Binder, G. Martino, P. Simon, G. Porraz, et N. Naudinot dir., Ressources lithiques, productions et transferts entre Alpes et Méditerranée, Paris, Société préhistorique française (coll. Séances, 5), p. 11-44.

VALDEYron N. (2008) - Sauveterrien et Sauveterriano : unité ou diversité du premier mésolithique en France méridionale et en Italie du nord, Pallas, 76, p. 247-259.

VISENTIN D. (2017) - Sauveterrian hunter-gatherers in Northern Italy and Southern France. Evolution and dynamics of lithic technical systems, Tesi di dottorato, Università degli Studi di Ferrara/Université Toulouse Jean Jaurès.
Guillaume Porraz

CNRS, ArScAn, AnTET, UMR 7041

Université de Paris Ouest-Nanterre guillaume.porraz@mae.u-paris10.fr

Antonin Tomasso TraceoLab/Préhistoire Université de Liège (Belgique)

Carlo Mologni

Université Côte d'Azur, CNRS, IRD, OCA Géoazur, UMR 7329, Sophia-Antipolis

Benjamin Audiard Université Côte d'Azur, CNRS CEPAM, UMR 7264, Nice

Leïla HoAREAu Université Côte d'Azur, CNRS CEPAM, UMR 7264, Nice

Marie-Anne Julien Unité Histoire Naturelle de l'Homme Préhistorique UMR 7194, MNHN, UPVD, Paris

Marco Padovan Dipartimento di Studi Umanistici Università degli Studi di Ferrara Ferrare (Italie)

Giorgia SARDELLI Dipartimento di Studi Umanistici Università degli Studi di Ferrara

Ferrare (Italie)

Patrick Simon

Musée d'Anthropologie Préhistorique de Monaco

Louise Purdue

Université Côte d'Azur, CNRS CEPAM, UMR 7264, Nice 